\title{
Age-based Estimation of Contact Lens Base Curve in Filipino Infants
}

\author{
Pamela Paulita P. Astudillo, MD, Roland Joseph D. Tan, MD, MS, \\ Romeo C. dela Cruz, MD and Marissa M. Valbuena, MD, MHPEd \\ Department of Ophthalmology and Visual Sciences, College of Medicine and Philippine General Hospital, University of the Philippines Manila
}

\begin{abstract}
Objective. To create an age-based formula to estimate the base curve needed for contact lens fitting using measured central corneal curvature (CCC) with a handheld auto keratometer from ophthalmologically normal infants.

Methods. This is a prospective cross-sectional study involving 70 ophthalmologically normal full-term infants aged 0 to 12 months. The infants were divided into four groups: 0-3 months, >3-6 months, $>6-9$ months and >9-12 months. CCC was measured with a handheld auto keratometer and was used to compute for the base curve. Differences in CCC between the four groups were measured and linear regression models were used for formula creation.

Results. The mean CCC was highest in group 1 at $45.5 \pm 2.4$ diopters (D). These values slowly decreased as age increased. A formula predictive of the base curve based on the infants age was derived using linear regression analysis. It predicted that for every month increase in age, there is a 0.063 millimeter increase in the base curve $(p<0.001)$.
\end{abstract}

Conclusion. Infant CCC is highest at birth and gradually decreases as age increases. The formula can predict the base curve needed for contact lens fitting in infants without the need for a handheld autokeratometer.

Keywords: base curve, central corneal curvature, contact lens, infants, Manila

\section{INTRODUCTION}

Corresponding author: Roland Joseph D. Tan, MD Department of Ophthalmology and Visual Sciences College of Medicine and Philippine General Hospital University of the Philippines Manila Taft Avenue, Ermita, Manila 1000, Philippines Email: rdtan@up.edu.ph
Proper optical correction in infants, including those who underwent extraction of cataractous lens, is important since amblyopia may ensue if overlooked. ${ }^{1}$ Available options for optical correction include the use of contact lens. In patients younger than seven months of age, contact lens use is the preferred method of optical correction, especially in post cataract surgery infants, since intraocular lens (IOL) implantation at this age was found to have more surgery-requiring complications. ${ }^{2}$ Contact lenses also offer less difference in the perceived size of images, good binocularity and better field of vision when compared to spectacle correction. ${ }^{3,4}$

However, a good contact lens fit is vital for successful optical correction. It facilitates proper tear exchange and allows good oxygen transmission to maintain corneal health. ${ }^{5}$ It also assures the stability of the contact lens in the eye, provides better quality of vision and results to decreased frequency of contact lens loss. A poor contact lens fit can cause corneal infection, dryness, opacification and vascularization secondary to corneal hypoxia that may even lead to loss of vision. ${ }^{1}$ An adequate fit is largely dependent on a properly measured base curve. 
Base curve is defined as the back surface of the contact lens which is dependent on and computed from the measured curvature of the central 3 millimeters of the cornea or the central corneal curvature (CCC). ${ }^{6}$ In cooperative and appropriately-sized patients, CCC can be easily measured using table-top manual or automated keratometers. However, in infants, a handheld auto keratometer is needed to obtain measurements. A handheld auto keratometer is an expensive ophthalmic equipment which not all ophthalmologists and hospitals can afford. This study aimed to determine if there is a relationship between the age of infants, CCC and the base curve, and if this relationship can be translated to a formula which estimates the base curve using only the age of the infant in the absence of a handheld auto keratometer.

\section{METHODS}

This prospective cross-sectional study was approved by the Philippine General Hospital Ethics Review Board. Well-baby infants born with a conceptional age of equal to or greater than 37 weeks and were 0 to 12 months of age seen at the Pediatric Outpatient Department of the Philippine General Hospital in 2011 were recruited and referred by Pediatric residents to the Pediatric Ophthalmology fellow for screening. Exclusion criteria included diagnosis of cataract, strabismus, micro- or macrophthalmia, microcornea, corneal scar and anterior segment dysgenesis, history of any type of intraocular surgeries, diagnosis with systemic problems and absence of consent from parents or guardians.

Current age during examination, conceptional age at birth, sex and ophthalmological history were collected from the parents of the participants. An ophthalmological examination was also performed by the pediatric ophthalmology fellow. A portable slit lamp was used to assess that the cornea and lens were normal. Infants who fit the inclusion criteria were divided into four age groups: 0-3 months (group 1), >3-6 months (group 2), >6-9 months (group 3) and $>9-12$ months (group 4). The central corneal curvature was then measured in diopters (D) by the pediatric ophthalmology fellow using a Nidek ARK30 hand-held keratometer (Nidek Co., Ltd, Japan). The measurements were performed with the infant in a sitting position or in a supine position with the keratometer held approximately 40 millimeters parallel to the infant's face (Figure 1). Although the lids were retracted, it was ensured that there was no undue pressure on the cornea that may affect the measurement. The infant was also allowed to blink before each measurement was obtained to prevent corneal dryness which can also negatively affect the measurement. The handheld auto keratometer automatically measured two axis of the central cornea 180 degrees apart which is standardly termed K1 (higher curvature) and K2. Three consecutive readings were obtained from each eye and the average was recorded. Readings from one eye of each infant were randomly selected and included in data analysis.

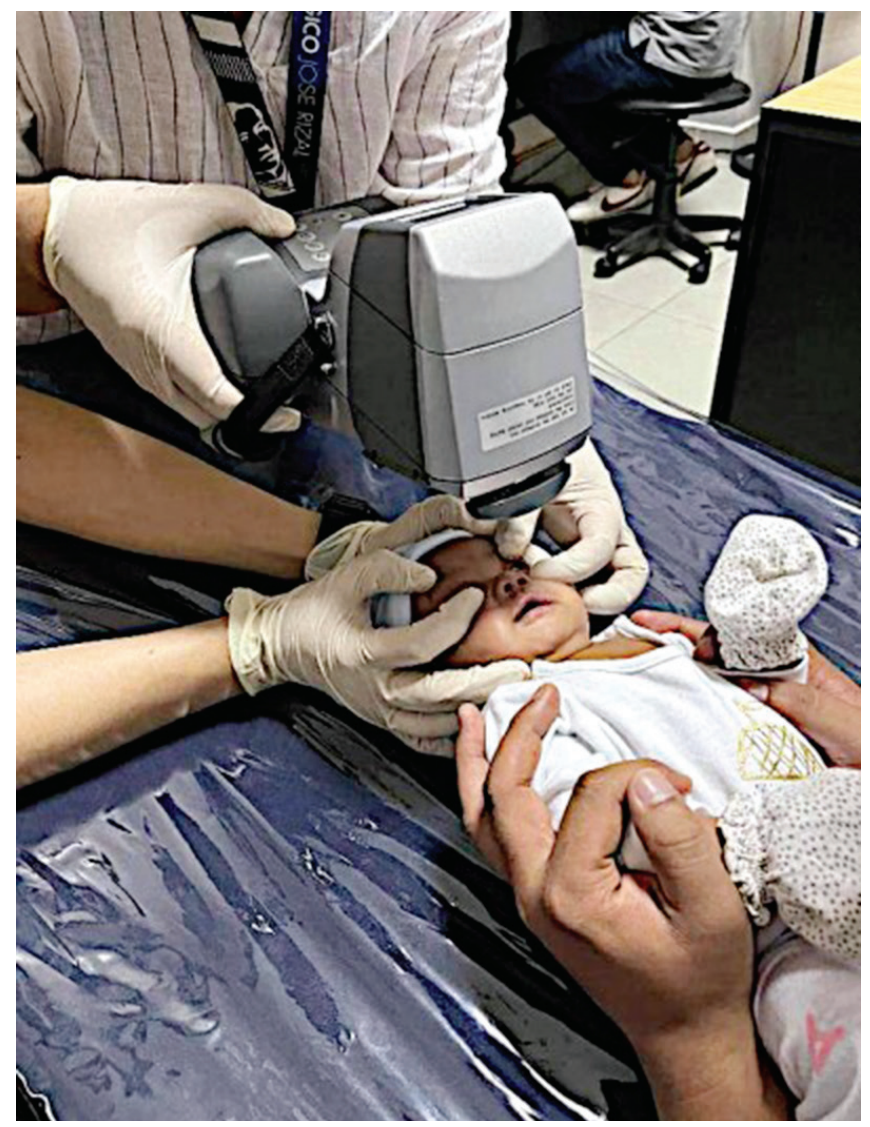

Figure 1. An infant undergoing measurement of the central corneal curvature using a handheld auto keratometer.

The contact lens base curve was then computed using the standard formula used for children and adults where the index of refraction of the cornea and index of refraction of air were constant values. ${ }^{7}$ The higher $\mathrm{CCC}$ measurement was represented by the $\mathrm{K} 1$ taken from the handheld auto keratometer. There is an inverse relationship between K1 and base curve.

Base Curve $=\frac{\text { Index of Refraction Cornea (1.3375) }- \text { Index of Refraction Air (1.000) }}{\text { Higher CCC measurement (in diopters) }} \times 1000$

Analysis of Variance (ANOVA) was utilized to test for variability of central corneal curvature (K1) and base curve measurements between eyes and across the age groups. Posthoc analysis using the Bonferroni method was performed for pairwise comparisons. Linear regression analysis was used to determine the base curve based on the infant's age in months.

\section{RESULTS}

Seventy infants referred from the Department of Pediatrics Well-baby clinic were included with 40 (57\%) being females. None were excluded. Of the 140 eye readings taken, only readings from the 70 eyes were included. 
Table 1. Average measurements of the eyes which was included in the analysis from each age group

\begin{tabular}{cccc} 
Age Group & No. of eyes & K1 (D) & Base Curve (mm) \\
\hline 1 & 25 & $45.45 \pm 2.39$ & $7.4 \pm 0.39$ \\
2 & 15 & $43.55 \pm 2.67$ & $7.8 \pm 0.57$ \\
3 & 15 & $41.77 \pm 1.68$ & $8.1 \pm 0.56$ \\
4 & 15 & $42.05 \pm 2.12$ & $8.1 \pm 0.54$ \\
\hline
\end{tabular}

Table 2. Post-hoc analysis of differences between K1 and base curve of each group

\begin{tabular}{cccccc}
\multirow{2}{*}{ Age Groups } & \multicolumn{2}{c}{ K1 (D) } & & \multicolumn{2}{c}{ Base Curve $(\mathrm{mm})$} \\
\cline { 2 - 3 } \cline { 5 - 6 } & Difference & p-value & & Difference & p-value \\
\hline 1 vs 2 & 1.9 & 0.38 & & 0.4 & 0.38 \\
1 vs 3 & 3.7 & $0.001^{*}$ & & 0.7 & $0.001^{*}$ \\
1 vs 4 & 3.4 & $0.001^{*}$ & & 0.7 & $0.001^{*}$ \\
2 vs 3 & 1.8 & 0.22 & & 0.3 & 0.21 \\
2 vs 4 & 1.5 & 0.47 & & 0.3 & 0.44 \\
3 vs 4 & 0.6 & 1.00 & & 0.0 & 1.00 \\
\hline
\end{tabular}

${ }^{*}$ statistically significant

ANOVA showed no significant variability in K1 (higher $\mathrm{CCC}$ measurement in diopters) between the right and left eyes of respective age groups. As such, one eye of each infant was randomly selected for data analysis (Table 1).

As age increased from group 1 to 4 , the CCC decreased while the base curve increased. The difference between each group was statistically significant $(\mathrm{p}<0.001)$. There were significant $\mathrm{K} 1$ and base curve differences between groups 1 and 3 and groups 1 and $4(\mathrm{p}<0.001)$ (Table 2$)$.

There was a gradual and steady increase in the base curve with increasing age (Table 1 and Figure 2). Using a linear regression model, the base curve was predicted with the following formula:

\section{Base Curve $=7.443+0.063$ (Age in months)}

wherein 7.443 is the $y$-intercept, which is the value of the base curve when age is equal to 0 months. The equation showed that every unit change of the infant's age in months corresponded to a $0.063 \mathrm{~mm}$ change in base curve value $(\mathrm{p}<0.001)$.

\section{DISCUSSION}

This study found that the mean base curve of infants increases as infants get older. This was similar to the findings in studies by Isenberg et al., Inagaki et al., Ehler et al. and Lightman and Marshall. ${ }^{8-11}$ The mean base curve in Lightman and Marshall et al.'s study was 7.6 millimeter in the first 6 months of life similar to this study. ${ }^{11}$ However, their mean base curve in 7 months was at 7.7 millimeters which stabilized at this value until 18 months of age. ${ }^{11}$ This study's mean base curve of infants was higher at 8.1 millimeter at 7 months onward and stabilized at this value

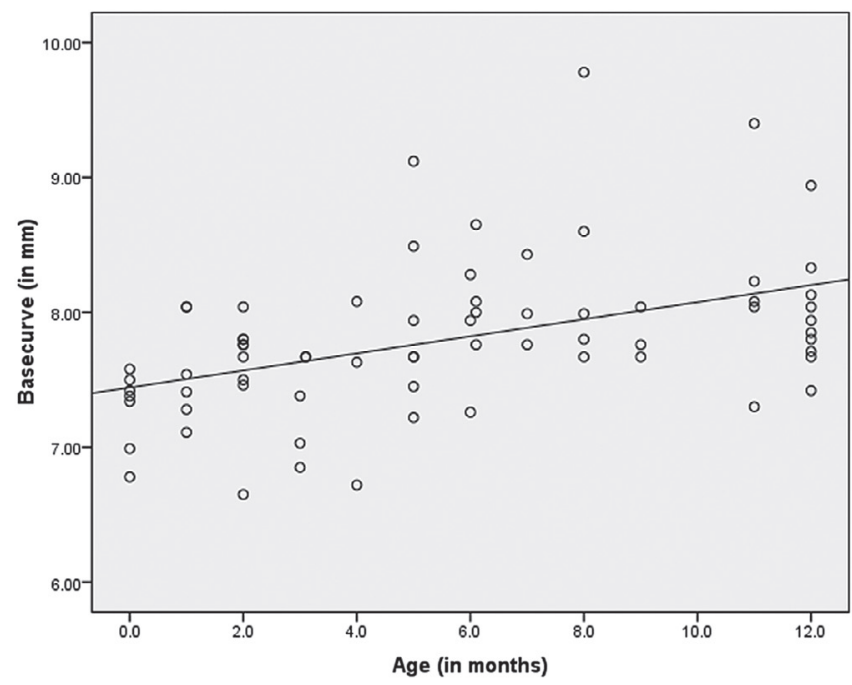

Figure 2. Scatter plot of infants age in months versus base curve measurements.

too up to the $12^{\text {th }}$ month of age. The stabilization of base curve after the $6^{\text {th }}$ month of life was also documented by Isenberg et al. and Capozzi. ${ }^{8,12}$ However, mean base curve at 0 month and 6 months in Isenberg et al. study were higher at 48.5 diopter and 43.0 diopter, respectively, compared to the findings of this study. ${ }^{8}$

This study provided an alternative option to estimate base curve for adequate contact lens fitting based on infant's age in the absence of an expensive handheld auto keratometer. However, the formula is not meant to replace actual measurement from an auto keratometer, if available. Lightman and Marshall had the same goal and measured the back optic radius of contact lens using silicone elastomer contact lens fitting. ${ }^{11}$ However, their technique was timeconsuming because several contact lenses with different base curve have to be tried before the right fit could be obtained. ${ }^{11}$ This study created a convenient and easy-to-use equation based on linear regression analysis of the measured CCC of healthy Filipino infants. This is the first study, to our knowledge, that attempted to do so as a response to the increasing need for contact lens fitting specially from the growing burden of congenital cataract in Filipino infants in the setting of a financially challenged health system. Handheld auto keratometers are not easily available in clinics and some hospitals, thereby making it difficult for ophthalmologists and optometrists to get good contact lens fit for infants. Even with the availability of handheld auto keratometer, some infants have to undergo repeated measurement under general anesthesia. Aside from the risk that repeated general anesthesia presents, most of our patients are unable to comply due to financial constraints as well.

The authors recommend additional studies with larger population to set normative data with acceptable maximum tolerable error as this study was only able to collect data 
that was sufficient to detect a significant pattern in base curve changes in infants aged $0-12$ months using a linear regression model and was made the basis of the formula to estimate base curves using infant's age in months. We further recommend to test its applicability to post-cataract surgery patients as data collected were based on ophthalmologically normal infants.

\section{CONCLUSION}

This study validated that, even in Filipinos, infant's $\mathrm{CCC}$ is highest at birth, and significantly decreases as age increases until the 6th month, after which it stabilizes. A formula was proposed to estimate the base curve needed for contact lens fitting in infants needing optical correction without the need for a handheld auto keratometer. Since the formula was based on ophthalmologically-normal infants, a study on its applicability to post-cataract surgery infants is recommended.

\section{Statement of Authorship}

All authors participated in the data collection and analysis and approved the final version submitted.

\section{Author Disclosure}

All authors declared no conflicts of interest.

\section{Funding Source}

This paper has no funding support.

\section{REFERENCES}

1. Neumann D, Weismann B, Isenberg S, Rosenbaum A, Bateman B. The effectiveness of daily wear contact lenses for the correction of infantile aphakia. Arch Ophthal. 1993; 111(7):927.

2. Plager D, Lynn M, Buckley E, Wilson M, Lambert S. Complications, adverse events, and additional intraocular surgery 1 year after cataract surgery in the infant aphakia treatment study. Ophthalmology. 2011; 118(12):2330-4.

3. Nelson L, Olitsky S. Harley's Pediatric Ophthalmology. Philadelphia: Wolters Kluwer; 2015.

4. Katsumi O, Miyanaga Y, Hirose T, Okuno H, Asaoka I. Binocular function in unilateral aphakia. Correlation with aniseikonia and stereoacuity. Ophthalmology. 1988; 95:1088-93.

5. Muntz A, Subbaraman L, Sorbara L, Jones L. Tear exchange and contact lenses: A review. J Optom. 2015; 8(1):2-11.

6. González-Cavada J, Corral O, Niño A, Estrella M, Fuentes J, Madrid-Costa D. Base curve influence on the fitting and comfort of the Senofilcon A Contact Lens. J Optom. 2009; 2(2):90-3.

7. Hua Y, Stojanovic A, Utheim T, Chen X, Ræder S, Huang J, et al. Keratometric Index obtained by Fourier-Domain Optical Coherence Tomography. PLoS One. 2015; 10(4):e0122441.

8. Isenberg SJ, Del Signore M, Chen A, Wei J, Christenson PD. Corneal topography of neonates and infants. Arch Ophthalmol. 2004; 122:1767-71.

9. Inagaki Y. The rapid change of corneal curvature in the neonatal period and infancy. Arch Ophthalmol. 1986; 104:1026-7.

10. Ehlers N, Sorensen T, Bramsen T, Poulsen EH. Central corneal thickness in newborns and children. Acta Ophthalmol (Copenh). 1976; 54:285-90.

11. Lightman JM, Marshall D, Jr. Clinical evaluation of back optic radius and curvature determination by age in pediatric aphakia due to congenital cataract fitted with a Silicone Elaster contact lens. Optom Vis Sci. 1996; 73:22-7.

12. Capozzi P, Morini C, Piga S, Cuttini M, Vadala P. Corneal curvature and axial length values in children with congenital/infantile cataract in the first 42 months of life. Inv Ophthal Vis Sci. 2008; 49:4774-8. 